\title{
Single $B$ cells reveal the antibody responses of rhesus macaques immunized with an inactivated enterovirus D68 vaccine
}

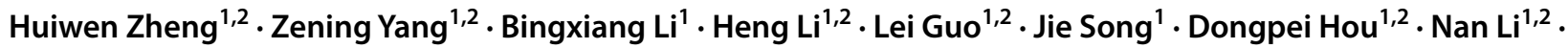 \\ Jinxi Yang ${ }^{1,2} \cdot$ Qiongwen $\mathrm{Wu}^{1,2} \cdot$ Ming Sun ${ }^{1} \cdot$ Longding Liu ${ }^{1,2}$
}

Received: 20 October 2019 / Accepted: 22 April 2020 / Published online: 28 May 2020

○) Springer-Verlag GmbH Austria, part of Springer Nature 2020

\begin{abstract}
Enterovirus D68 (EV-D68) infection may cause severe respiratory system manifestations in pediatric populations. Because of the lack of an effective preventive vaccine or specific therapeutic drug for this infection, the development of EV-D68-specific vaccines and antibodies has become increasingly important. In this study, we prepared an experimental EV-D68 vaccine inactivated by formaldehyde and found that the serum of rhesus macaques immunized with the inactivated EV-D68 vaccine exhibited potent neutralizing activity against EV-D68 virus in vitro. Subsequently, the antibody-mediated immune response of B cells elicited by the inactivated vaccine was evaluated in a rhesus monkey model. The binding activity, in vitro neutralization activity, and sequence properties of 28 paired antibodies from the rhesus macaques' EV-D68-specific single memory B cells were analyzed, and the EV-D68 VP1-specific antibody group was found to be the main constituent in vivo. Intriguingly, we also found a synergistic effect among the E15, E18 and E20 monoclonal antibodies from the rhesus macaques. Furthermore, we demonstrated the protective efficacy of maternal antibodies in suckling C57BL/6 mice. This study provides valuable information for the future development of EV-D68 vaccines.
\end{abstract}

\section{Introduction}

Enterovirus D68 (EV-D68) is a picornavirus that belongs to the species Enterovirus D [1]. In August 2014, a large outbreak of EV-D68 occurred in the United States that caused severe respiratory illness [2]. Recently, EV-D68 was found

Handling Editor: Ana Cristina Bratanich.

Huiwen Zheng and Zening Yang contributed equally to this work.

Electronic supplementary material The online version of this article (https://doi.org/10.1007/s00705-020-04676-6) contains supplementary material, which is available to authorized users.

Ming Sun

sunming@imbcams.com.cn

$\triangle$ Longding Liu

longdingl@gmail.com

1 Institute of Medical Biology, Chinese Academy of Medical Sciences, Peking Union Medical College, Kunming 650118, China

2 Key Laboratory of Systemic Innovative Research on Virus Vaccine, Chinese Academy of Medical Sciences, Beijing, China to be locally prevalent in Europe, Asia and North America [3-5]. In addition to causing severe respiratory disease, EV-D68 has also been reported to be associated with cranial nerve dysfunction $[2,6]$. However, there is neither a specific therapeutic drug for this infection nor a prophylactic vaccine that can prevent it. Although previously developed EV-D68 virus-like particle (VLP) vaccines and inactivated vaccines can protect suckling mice from lethal EV-D68 challenge, the mechanism of action of these vaccines has not been elucidated [7, 8].

Neutralizing antibody (Nab) production is the main immunological evaluation index for inactivated vaccines. For example, the Nab titer has been successfully applied to evaluate the effectiveness of inactivated vaccines against enterovirus A71 (EV-A71) and poliovirus [9-11]. However, Nab titers do not completely reflect the vaccine-induced systemic immunological response. Several studies have shown that multiple types of antibodies, including neutralizing antibodies, nonneutralizing antibodies, and antibodies against different epitopes, cooperatively participate in the vaccine-induced immune response and control viral infection [12-14]. For example, HIV, Ebola virus, and H7N9 viruses can all elicit a variety of antibodies to control infection $[12,13,15]$. Therefore, exploring the distribution of 
antibody lineages induced by a vaccine in the body and the epitope-recognizing characteristics of different antibodies can help us better understand the vaccine-induced immune response. In this study, the single $B$ cell isolation technique was applied to investigate the evolution of the EV-D68-specific antibody lineage in a rhesus macaque. At the same time, the binding and neutralization characteristics of EVD68-specific antibodies were used to assess the effectiveness of the EV-D68 vaccine in a preclinical evaluation based on the diversity of rhesus macaque $\mathrm{B}$ cells and the antibody spectrum.

In our previous study, we found that rhesus macaques naturally infected with EV-D68 produced Nabs mainly against EV-D68 VP1 in vivo [16]. We isolated Nab A6-1, but this antibody exhibited only a moderate neutralizing effect [16]. In the current study, we systematically analyzed the types and characteristics of antibodies induced by a formaldehydeinactivated EV-D68 vaccine in rhesus monkeys and found that monoclonal antibodies (mAbs) against the EV-D68 VP1 protein were the main types of antibodies elicited by this experimental vaccine. When applied in combination with other antibodies, the neutralization effect of such antibodies may be enhanced. Thus, the inactivated vaccine can effectively elicit host antibody responses against the major antigen of EV-D68. These results also indicate that the analysis of antibody spectrum properties provides a new perspective for evaluating EV-D68 vaccines.

\section{Materials and methods}

\section{Viruses and cells}

The EV-D68 KM strain (GenBank ID: MG991260) used in the present study, was obtained from the Institute of Medical Biology (IMB), Chinese Academy of Medical Sciences (CAMS). The EV-D68 Fermon strain (GenBank ID: AY426531.1) was from the American Type Culture Collection (ATCC). The concentration of the EV-D68 virus stock was $5.5 \mathrm{log}$ cell culture infectious doses $\left(\mathrm{CCID}_{50}\right) / \mathrm{ml}$. This batch of virus stock was then preserved for follow-up experiments. The source of the Vero cells used was the ATCC.

\section{Preparation of the experimental inactivated EV-D68 vaccine and animal immunization protocol}

The EV-D68 KM strain was inoculated onto a monolayer of Vero cells. The EV-D68 viral suspension was harvested at 72 hours postinfection at a multiplicity of infection (MOI) of 0.1 at $37^{\circ} \mathrm{C}$. Then, the harvested viruses were centrifuged at $3000 \times g$ for 30 minutes, and the supernatant was collected. The EV-D68 viral suspension was inactivated with formaldehyde at a concentration of 1:4000 and then maintained at $37{ }^{\circ} \mathrm{C}$ for 7 days. After ultrafiltration and concentration using a 100,000 molecular weight cutoff (MWCO) filter, the inactivated virus was purified by Sepharose 6FF chromatography, and the protein concentration of the viral antigen was determined using a BCA Protein Assay Kit (Thermo Fisher, Rockford, IL 61101, USA.) [17]. For stock production, $1 \mathrm{mg}$ of aluminum hydroxide $\left(\mathrm{Al}(\mathrm{OH})_{3}\right)$ adjuvant per $\mathrm{ml}$ was added to the purified viral suspension. Each $0.5 \mathrm{ml}$ of the final vaccine product contained $8 \mu \mathrm{g}$ of protein antigen. The vaccine samples were used in subsequent experiments after confirmation of inactivation.

Two healthy 6-month-old rhesus monkeys (Macaca mulatta) (ID: 15239, 15083) were inoculated with the experimental EV-D68 inactivated vaccine at 0 and 28 days postvaccination. Blood samples were collected from the animals at $0,14,28$, and 56 days post-vaccination (Fig. S1). All animal experiments were approved by the Institutional Animal Care and Use Committee (IACUC) of tIMB, CAMS. Before starting the study, it was confirmed that the two monkeys did not carry anti-EV-D68 antibodies.

\section{Viral challenge of suckling mice}

Six-week-old female C57BL/6 mice were inoculated with the EV-D68 inactivated vaccine or PBS at 4-week intervals, which was followed by mating with the male mice at two weeks after the final immunization [18]. After the newborns were delivered, the two-day-old suckling mice were infected with EV-D68 through intracranial administration with $5 \times 10^{4} \mathrm{CCID}_{50}$ of the EV-D68 KM strain. All challenged mice were monitored daily for survival for 15 days.

\section{Antigens and peptides}

The EV-D68 full-length structural protein antigens VP1, VP2 and VP3 were prepared previously and obtained using the prokaryotic expression method. Subsequently, peptides containing the main neutralizing structural regions, namely, the BC loop, DE loop and GH loop of the VP1 protein, were synthesized as described in a previous report [16].

\section{Sorting of single EV-D68-specific memory B cells by flow cytometry}

PBMCs were stained with $100 \mu 1$ of PBS containing a mixture of antibodies against CD20 (PE; 2H7), CD27 (FITC; M-T271), and EV-D68-APC, and the cells were incubated for 30 minutes at room temperature in the dark. Except for the anti-EV-D68-APC antibody, all antibodies used in flow cytometry experiments were purchased from BD Biosciences, and the anti-EV-D68-APC conjugate was prepared according to the protocols provided by the manufacturer (Innova Bioscience Ltd., USA). The flow 
gating and sorting process has been described previously [16]. The flow cytometry data were analyzed using FlowJo V10 software (FlowJo, Ashland, OR). The antigen integrity of labeled EV-D68 virus (EV-D68-APC) was verified by SDS-PAGE and Western blot (WB) with serum from EV-D68-immunized rhesus monkeys. The SDS-PAGE and Western blot assays have been described previously [16].

\section{ELISPOT assay}

The EV-D68 structural proteins VP1, VP2 and VP3 and the whole EV-D68 virus were selected as stimuli and used to detect EV-D68-specific IFN-secreting cells or EV-D68-specific IgG-producing B cells. A monkey ELISPOT assay was performed according to the manufacturer's instructions. In brief, precoated plates were blocked with cell culture medium containing $10 \%$ fetal bovine serum (FBS) for 1 hour at room temperature. Subsequently, PBMCs $\left(5 \times 10^{5}\right.$ cells/well $)$ were added to the 96 -well plate in triplicate, followed by incubation with a stimulant at a concentration of $10 \mu \mathrm{g} / \mathrm{ml}$. Next, the plate was placed at $37{ }^{\circ} \mathrm{C}$ for 48 hours. After incubation, the cells were removed, and the color was developed until the spots were clearly visible. Then, an ELISPOT plate reader was used to count the spot-forming cells (SFCs) [17].

\section{Amplification of EV-D68-specific mAb genes}

The monkey Ig heavy chain and light chain genes were amplified by reverse transcription PCR and nested PCR. All PCR conditions, reaction temperatures, and primers have been described previously [16].

\section{Antibody expression and testing}

IgG expression vectors (pCMV-Rh) with a leader peptide and IGH, IGK, and IGL constant regions were used to express our mAbs. Then, the EV-D68 mAbs were expressed in $293 \mathrm{~T}$ cells and purified using protein A beads (Thermo Fisher Scientific, USA). 293T cells were cotransfected with the heavy chain plasmid and light chain plasmid at a molar ratio of 3:2 using Lipofectamine 2000 (Thermo Fisher Scientific, USA) for transient expression. After harvesting the supernatants from expressing cells, the antibodies were purified using protein A beads. Finally, the purified EV-D68 mAbs were detected using HRP-conjugated rabbit anti-human $\operatorname{IgG}(1: 5000$, Abcam) at room temperature. The protein bands were visualized using an ECL Western blot detection kit [16].

\section{Neutralization assays}

Monkey serum neutralizing titers were determined by standard microneutralization assays as described previously [16]. The single or combined mAb neutralization effectiveness of the mAbs was assessed as follows [13]: In vitro neutralization activity of antibodies was evaluated using a microneutralization assay. To assess the $\mathrm{IC}_{50}$ titers of single mAbs, $50 \mu \mathrm{l}$ of $\mathrm{mAb}$ at various concentrations was incubated with $50 \mu \mathrm{l}$ of the EV-D68 KM strain or Fermon strain $\left(100 \mathrm{CCID}_{50}\right)$ for 1 hour at $37^{\circ} \mathrm{C}$. To assess the $\mathrm{IC}_{50}$ titers of a pair of mAbs, antibodies were mixed at the indicated ratio. The paired $\mathrm{mAbs}$ were then mixed with $50 \mu 1$ of the EV-D68 KM strain or Fermon strain $\left(100 \mathrm{CCID}_{50}\right)$ for 1 hour at $37{ }^{\circ} \mathrm{C}$. Next, the antibodyvirus mixture was added to the Vero cell monolayer and incubated for 2 hours, followed by supplementation of the DMEM culture medium with $1 \%$ FBS. The 96-well plates were then placed in a $37{ }^{\circ} \mathrm{C}$ incubator for 72 hours and the $\mathrm{IC}_{50}$ was determined using a 3-(4,5-dimethylthiazol2-yl)-5-(3-carboxymethoxyphenyl)-2-(4-sulfophenyl)$2 \mathrm{H}$-tetrazolium (MTS) assay, and the absorbance was measured upon excitation at $490 \mathrm{~nm}$. The neutralization efficiency of each sample was calculated as follows: percent neutralization $=\left\{1-\left[\left(\mathrm{OD}_{490}\right.\right.\right.$ of each sample $-\mathrm{OD}_{490}$ of the cells treated with virus only) / ( $\left(\mathrm{OD}_{490}\right.$ of untreated cells $-\mathrm{OD}_{490}$ of the cells treated with virus only)] $\} \times 100$. The $\mathrm{IC}_{50}$ was defined as the concentration of $\mathrm{mAbs}$ that inhibited cell death by $50 \%$. The final $\mathrm{IC}_{50}$ value for each $\mathrm{mAb}$ was calculated as described previously [16].

\section{Enzyme-linked immunosorbent assay (ELISA)}

The binding capacity of monkey serum or mAbs was determined by methods established in previous work. Briefly, ELISA plates were coated overnight at $4{ }^{\circ} \mathrm{C}$ with the antigens VP1, VP2, VP3, EV-D68 virus, BC loop peptide, DE loop peptide, or GH loop peptide at a concentration of $0.2 \mu \mathrm{g} / \mathrm{well}$. The plates were then blocked with PBST containing 10\% BSA for 2 hours at room temperature. Next, the purified $\mathrm{mAbs}$ were added to the plates, which were incubated for 2 hours at room temperature. Subsequently, an HRP-conjugated detection antibody (Abcam, USA) was added at a 1:20,000 dilution for 1 hour. The $\mathrm{OD}_{450}$ value was then measured using a continuous-wavelength microplate reader. Samples with $\mathrm{OD}_{450}$ values $>2.1$ times the $\mathrm{OD}_{450}$ of the negative control sample were deemed to exhibit positive binding.

\section{Phylogenetic analysis of antibody sequences}

The heavy-chain and light-chain (kappa and lambda) germline distributions of the mAbs were analyzed using 
IgBLAST (https://www.ncbi.nlm.nih.gov/igblast/). We constructed heavy-chain, kappa-chain and lambda-chain phylogenetic trees using known mAb sequences. The mAb evolutionary tree was constructed using MEGA 5.0. All phylogenetic trees were constructed by the neighbor-joining method with 1000 bootstrap replicates.

\section{Statistical analysis}

All line charts and bar charts were generated using GraphPad Prism 5. All data were analyzed using SPSS 19.0 software, and $p<0.05$ was considered statistically significant.

\section{Results}

\section{Humoral and cellular responses to VP1 elicited by the experimental inactivated EV-D68 vaccine}

The virus preparation $\left(10^{5.5} \mathrm{CCID}_{50} / \mathrm{ml}\right)$ was incubated with formalin at a concentration of $1: 4000$ at $37{ }^{\circ} \mathrm{C}$ for 7 days, and a virus titration assay was carried out to verify vaccine inactivation (Fig. S2). Then, two monkeys were immunized with the experimental vaccine. Twenty-eight days after the first immunization dose, the two monkeys had neutralizing antibody titers of 7.3 and 14.5 against the EV-D68 KM strain. After the booster injection, the titers increased to 31.8 and 63.7 (Fig. 1a). The antibodies showed a binding preference for VP1 and the EV-D68 whole virus over VP2 and VP3 (Fig. 1b). To evaluate the EV-D68-specific cellular response, we carried out an IFN- $\gamma$ ELISPOT assay on samples collected from EV-D68-immunized rhesus monkeys (Fig. 1c, Fig. S3). Stimulating PBMCs with the EV-D68 VP1, VP2, and VP3 structural proteins or the whole virus revealed that the specific T-cell IFN- $\gamma$ response in immunized monkeys was elicited by the EV-D68 whole virus and by the EV-D68 VP1, VP2, and VP3 structural proteins $(p<0.05)$. In addition, we evaluated EV-D68-specific IgGsecreting B cells. VP1, VP2, VP3, and the EV-D68 virus all effectively elicited an EV-D68-specific IgG-secreting B cell response $(p<0.05)$ (Fig. 1d, Fig. S3). However, compared with VP2 and VP3, EV-D68 and VP1 elicited stronger cellular responses. These results indicated the ability of this experimental EV-D68 vaccine to elicit humoral and cellular immune responses in monkeys.

\section{Protection of suckling mice born to mothers immunized with inactivated EV-D68 vaccine against lethal EV-D68 infection}

Six female C57BL/6 mice were immunized with the inactivated EV-D68 experimental vaccine. At two weeks after the second immunization, serum samples were collected and analyzed using a microneutralization assay. Compared with that of the control serum $(<4)$, the geometric mean titer (GMT) of all EV-D68-immunized mouse sera was greater than or equal to 45.3 (Fig. 2, Table S1). The two female mice (A and F) with the highest GMT (128) were then selected to mate with the male mice (Table S1). Two-day-old suckling mice born to immunized mothers $\mathrm{A}$ and $\mathrm{F}$ were infected with the KM strain. During a 15-day observation period, suckling mice born to unimmunized mothers (PBS group) displayed body weight loss (data not shown) beginning at 3 days postinfection, and $80 \%$ of these mice died by 15 days postinfection (Fig. 2). In contrast, $100 \%$ of the neonatal mice from the mothers immunized with inactivated EV-D68 vaccine survived without any signs of disease (Fig. 2). These results indicate that the inactivated EV-D68 vaccine can induce EV-D68-specific mAbs in suckling mice.

\section{EV-D68-specific memory B cells sorted from immunized animals}

For sorting of single EV-D68-specific memory B cells from EV-D68-immunized rhesus monkeys, blood specimens were collected from two monkeys after vaccination. In this study, the EV-D68 virus conjugated with APC fluorescein was used to screen EV-D68-specific B cells, and the integrity of the main structural antigens of VP1, VP2, and VP3 was verified as shown in Fig. S4. At 28 days after the primary immunization, a population of memory B cells $\left(\mathrm{CD} 20^{+} \mathrm{CD} 27^{+} \mathrm{EV}-\mathrm{D} 68^{+}\right)$was clearly identified as $12.5 \%$ of the total CD20 ${ }^{+} \mathrm{CD} 27^{+} \mathrm{B}$ cells in one monkey (Fig. 3a) and $28.6 \%$ in the other (Fig. 3b), indicating that the inactivated EV-D68 vaccine effectively elicited a specific B-cell immune response.

\section{Sequence analysis of vaccine-induced antibodies}

We performed cDNA synthesis and nested PCR amplification according to a previously established method [16]. The lengths of positive fragments, including those of IGH VDJ, IGL VJ, and IGK VJ, ranged from approximately $300 \mathrm{bp}$ to $500 \mathrm{bp}$. The heavy-chain gene was successfully amplified from $29.9 \%$ of the samples (109 of 364), and 18.7\% (68 of 364) were positive for light-chain amplification. In total, the cells in 28 wells produced both heavy and light chains, which can be used to express potentially functional monoclonal antibodies.

The IGHV, IGKV and IGLV subgroup distribution of the mAbs and the CDR3 length of IG cloned from different single memory B cells were analyzed. For the Ig heavychain variable region (IGHV), IGHV3 accounted for $61.5 \%$ of the total subgroups, followed by IGHV1 (23.1\%), IGHV4 (11.8\%) and IGHV7 (3.8\%). For the Ig kappa-chain variable region (IGK), IGKV1 and IGKV3 both accounted for the 

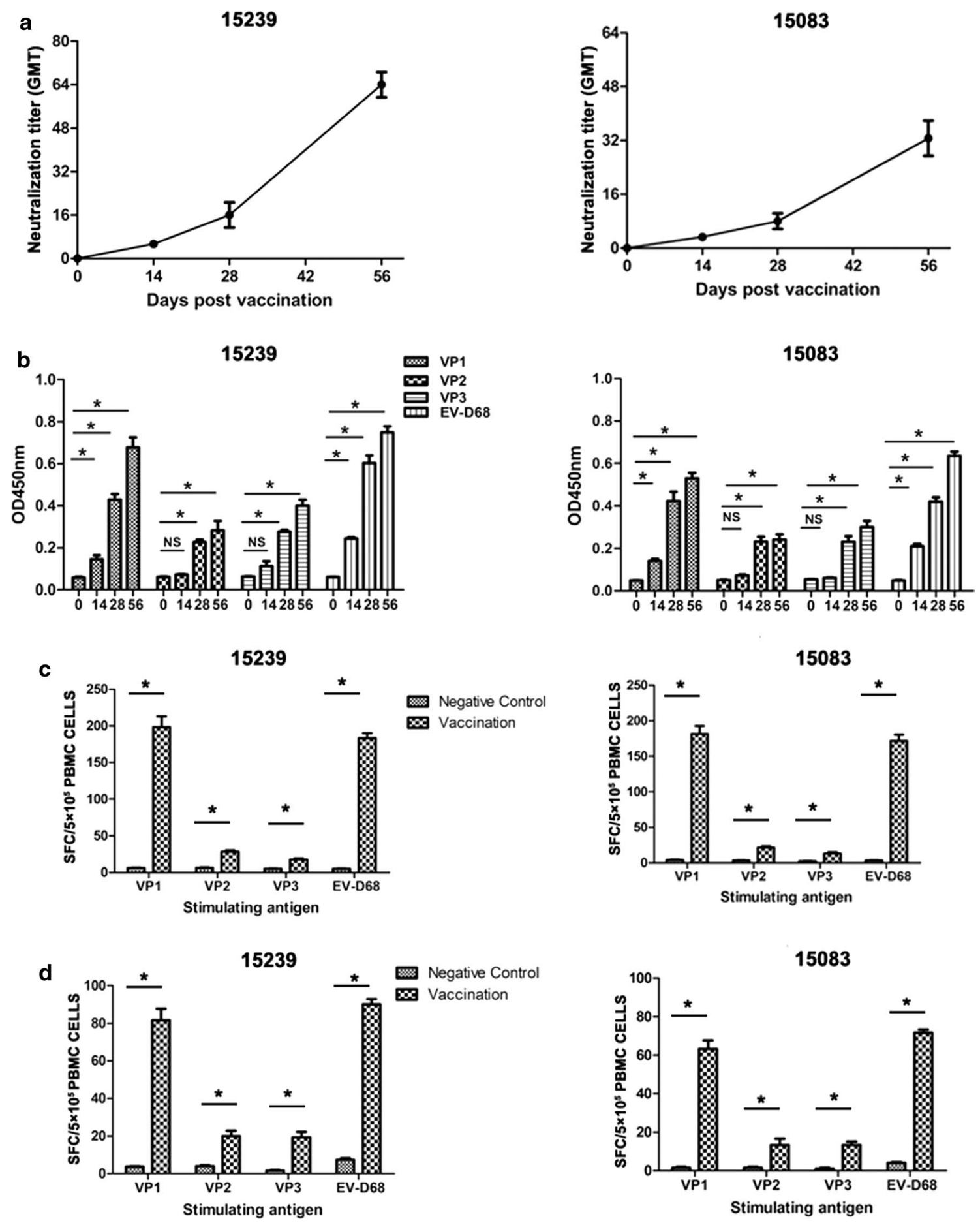

Fig. 1 Serological analysis of rhesus macaques after EV-D68 vaccination. (a) EV-D68-neutralizing antibody titers of two immunized animals. The neutralization $\mathrm{Ab}$ titer was the reciprocal of the highest serum dilution that inhibited 50\% of the viral CPE. (b) Analysis of the ability of serum antibodies to bind viral structural proteins

largest proportion (37.5\%) of the subgroups, followed by IGKV4 and IGKV2 (16.7\% and 8.3\%, respectively). The Ig lambda-chain variable region (IGL) was composed mainly
(VP1, VP2, and VP3) or the whole virus at $0,14,28$, and 56 days after vaccination. (c) EV-D68-specific IFN- $\gamma$ response to EV-D68 as determined by ELISPOT assay. (d) IgG-secreting B-cell response to EV-D68 as determined by ELISPOT assay. *, $p<0.05$

of IGLV3 (85.7\%) and IGLV1 (14.3\%). In addition, all of the heavy-chain CDR3 sequences contained more than nine amino acids and consisted of a small number of relatively 


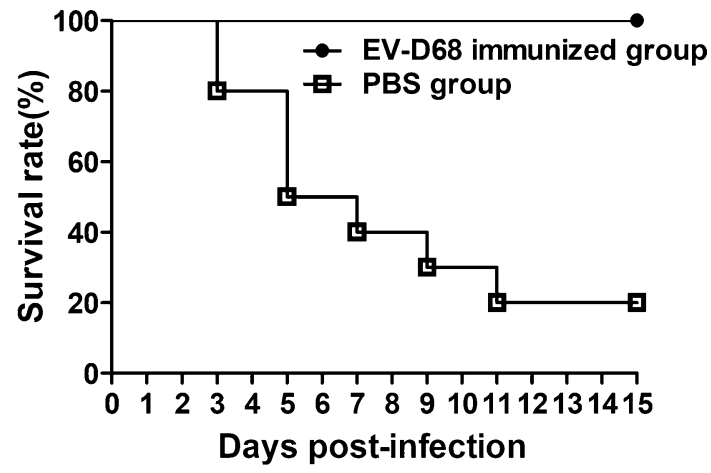

Fig. 2 Survival of neonatal mice born to female mice immunized with the inactivated EV-D68 vaccine. The suckling mice of the EVD68-immunized group $(n=12)$ or the PBS group $(n=10)$ were observed daily for survival for 15 days

long CDR3 sequences (>19 aa). A majority of the IGK CDR3 sequences were nine amino acids in length, and the V-lambda sequences were 12 amino acids in length (Fig. 4a). The virus-specific subgroup was strongly induced to form a particular IGHV, IGKV and IGLV germline in the primary immunized rhesus macaque. The closest $\mathrm{V}(\mathrm{D}) \mathrm{J}$ germline genes for the heavy and light chains of the $28 \mathrm{mAbs}$ are shown in Table $\mathrm{S} 2$. These $\mathrm{mAb}$ heavy and light chains all originated from different germline genes. The nucleotide sequence identity values for the heavy chain ranged from $70.6 \%$ to $97.2 \%$. However, the light chain showed less somatic hypermutation than the heavy chain, with values ranging from $80.5 \%$ to $99.3 \%$ (Table S2). Compared with the prevaccination results (Fig. S5), the subgroup distributions of IGHV, IGKV, IGLV and CDRH3 lengths were found to be changed in the rhesus macaques after vaccination (Fig. 4a). We concluded that the antibody gene was rearranged in response to inoculation with the inactivated EV-D68 vaccine, resulting in changes in the representative antibody germline and the CDR3 length.

\section{Binding of $m A b s$ to the EV-D68 VP1 protein}

A genetic evolutionary cluster analysis divided the heavy chains into A1, A2, B1, and B2 clusters. The light chain (including kappa and lambda) of the EV-D68-specific antibodies was divided into clusters A and B (Fig. 4b). An EV-D68 VP protein-binding assay showed that E4, E15, E16, E18, E20, and E21 were able to bind to EV-D68 VP1, VP2, and VP3 (Fig. 5a, b, and c), with strong binding to VP1 and weak binding to VP2 or VP3. These antibodies belonged to the $\mathrm{A} 1, \mathrm{~A} 2$, and $\mathrm{B} 2$ clades according to heavy-chain gene clustering. The BC, DE and GH loops of EV-D68 VP1 may contain neutralizing sites [19]. Therefore, BC, DE and GH loop peptide-scanning experiments for VP1 were carried out with the six antibody pairs. The antibodies E4, E15 and E21 of cluster A1 reacted strongly with peptides 12 and 13 of the DE loop but weakly with the other peptides (Fig. 5d). The antibodies E18 and E20 of cluster A2 reacted with peptide 20 of the GH loop, but no binding was observed with the remaining peptides (Fig. 5e). The mAb E16 of B2 showed only weak binding to peptides 12 and 13 of the DE loop (Fig. 5f). Collectively, these results demonstrate that the paired antibodies isolated from monkeys vaccinated with the EV-D68 experimental vaccine mainly recognized the VP1 structural protein.

\section{Single and cooperative neutralization characteristics of $\mathrm{mAbs}$ targeting EV-D68 in vitro}

We next tested the ability of these six candidates to neutralize EV-D68. The neutralization assay revealed that $\mathrm{mAbs}$ E15 of cluster A1 and mAbs E18 and E20 of cluster A2 had different neutralization effects, and their $\mathrm{IC}_{50}$ values $(\mu \mathrm{g} / \mathrm{ml})$ were $1.69,4.20$, and $5.03 \mu \mathrm{g} / \mathrm{ml}$, respectively, for the $\mathrm{KM}$ strain and 2-10 $\mu \mathrm{g} / \mathrm{ml}$ for the Fermon strain (Fig. 6a and b). The other three mAbs, E4 and E21 of cluster A1 and E16 of cluster B2, showed weak neutralization ability, with $\mathrm{IC}_{50}$ values greater than $20 \mu \mathrm{g} / \mathrm{ml}$ (data not shown). Therefore, we did not study the mAbs E4, E21 and E16 further. For binding to EV-D68 VP1 of the Fermon or KM strain, the $\mathrm{EC}_{50}$ values of E15 were $1.11 \mu \mathrm{g} / \mathrm{ml}$ and $2.26 \mu \mathrm{g} / \mathrm{ml}$, respectively; thus, it showed greater potency than E18 and E20 (1.80 and $1.84 \mu \mathrm{g} /$ $\mathrm{ml}$, or 3.65 and $4.31 \mu \mathrm{g} / \mathrm{ml}$ ) (Fig. $6 \mathrm{c}$ and d). We then investigated whether different $\mathrm{mAbs}$ exhibit cooperative reactivity when combined $[13,20]$ and found that the mean $\mathrm{IC}_{50}$ value of $\mathrm{E} 15$ against the KM strain decreased (from 1.69 to 0.82 and 1.19) in the presence of E18 and E20, respectively, at a fixed concentration of $20 \mu \mathrm{g} / \mathrm{ml}$ (Table 1, Fig. 7a and b). Moreover, the mean $\mathrm{IC}_{50}$ value of E15 decreased (from 1.69 to 1.17 and 1.40) in the presence of the original concentration of E18 $(4 \mu \mathrm{g} / \mathrm{ml})$ and E20 $(5.0 \mu \mathrm{g} / \mathrm{ml})$, respectively (Table 1, Fig. 7c and d). We also verified the synergistic neutralization effect against the Fermon strain. However, the synergistic neutralization effect against the Fermon strain was weaker than that against the KM strain (Fig. 7e, f, g, and $\mathrm{h}$, Table 1). The above results demonstrate that various antibodies from different clusters have different neutralization capacities, and synergistic neutralization activity between antibodies was observed.

\section{Discussion}

EV-D68 can cause serious infection outcomes [21-23], and there are currently no effective preventive vaccines or therapeutic drugs. Although EV-D68 VLPs and inactivated vaccines have been reported previously, the mechanism of the immune response associated with these vaccines remains 
a Od

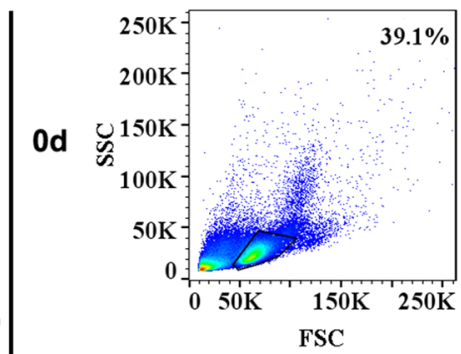

15239

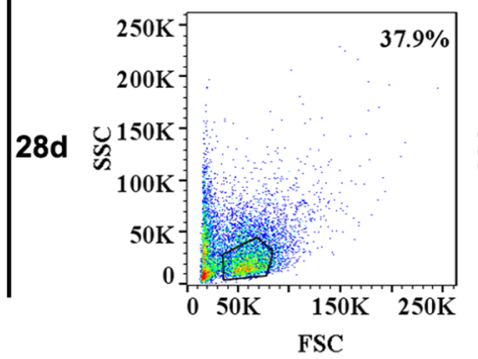

b

15083

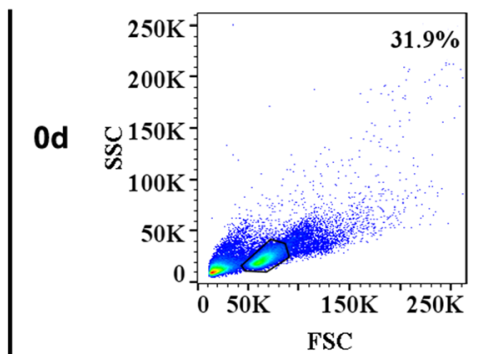

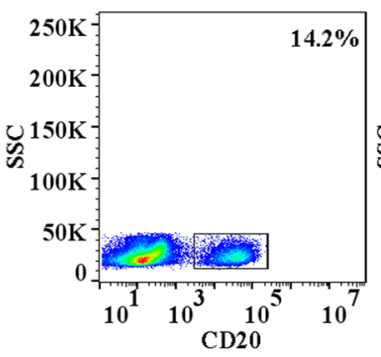
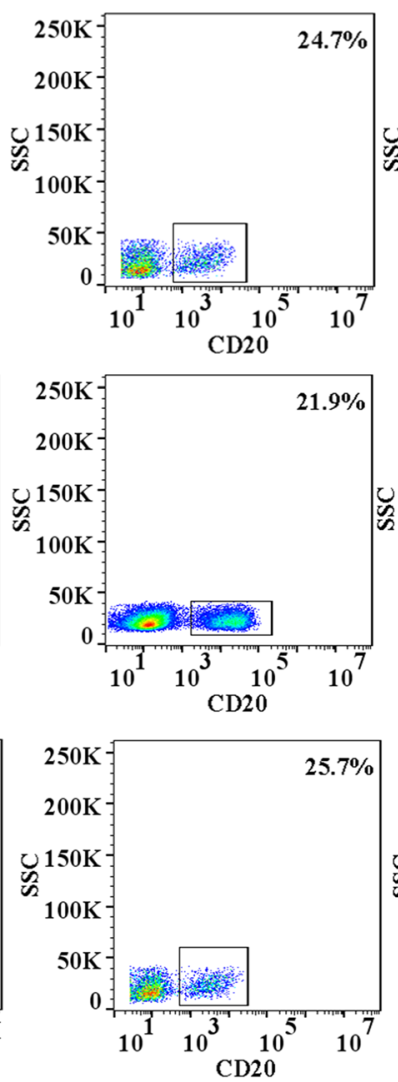
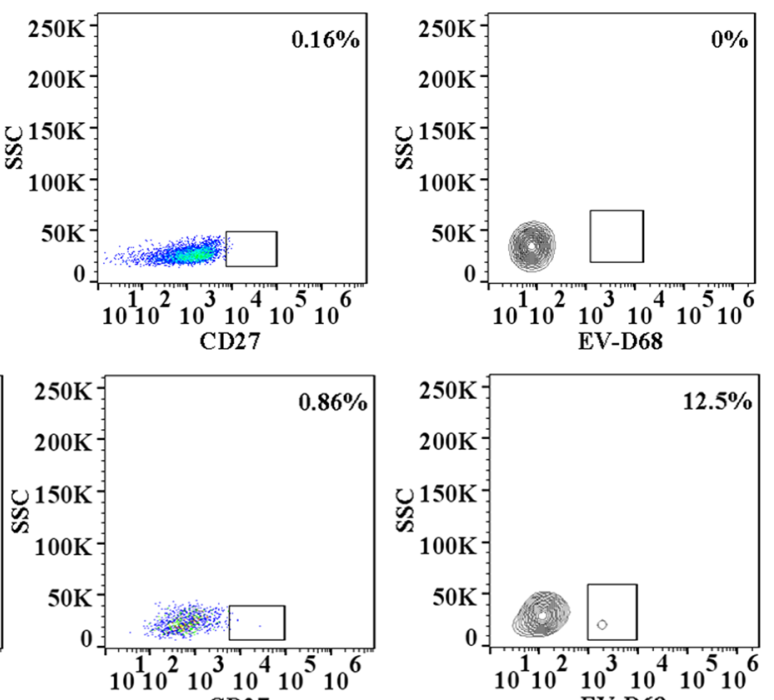

CD27
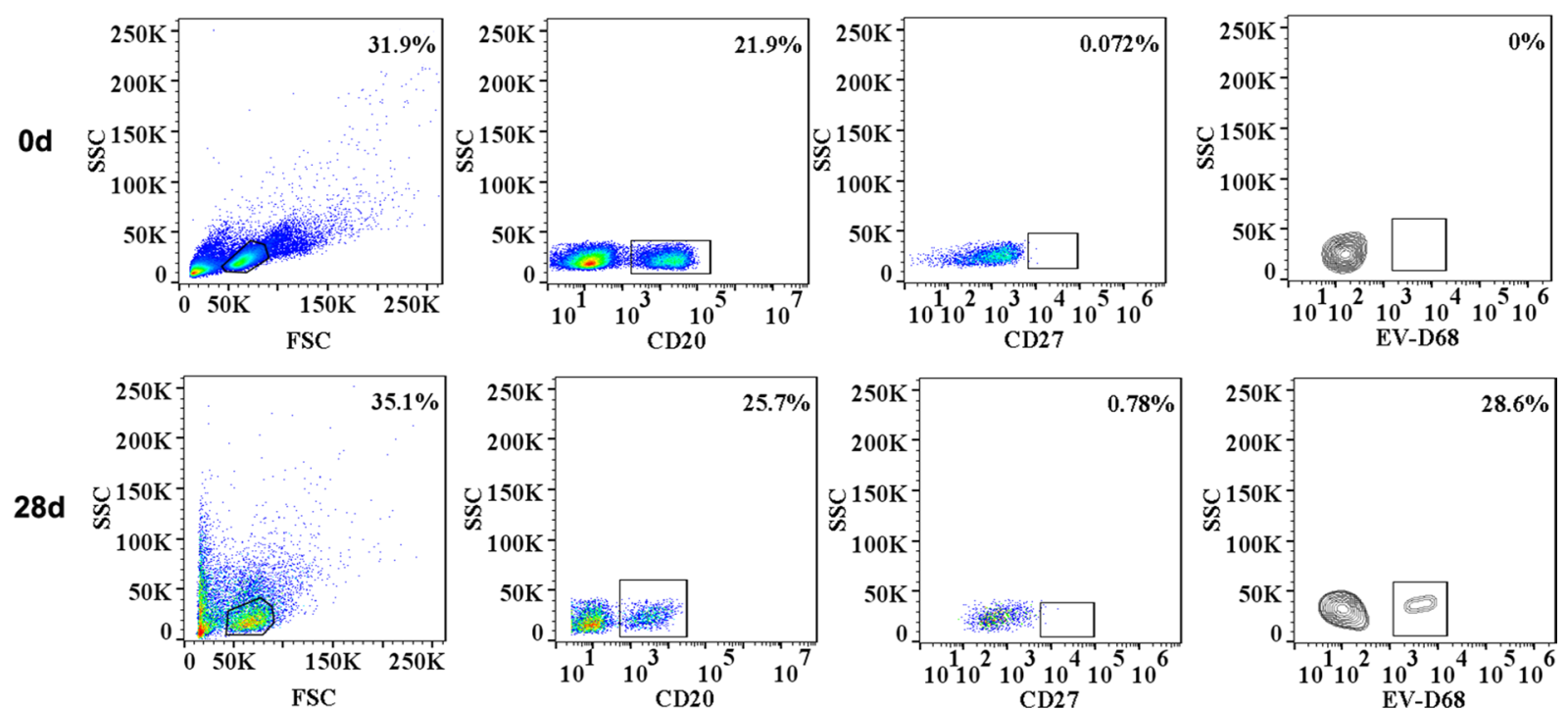

Fig. 3 Sorting of single EV-D68-specific memory B cells by FACS. PBMCs were stained with an antibody cocktail including anti-CD20 (PE), anti-CD27 (FITC), and anti-EV-D68 (APC) antibodies. Single EV-D68-specific memory $\mathrm{B}$ cells $\left(\mathrm{CD} 20^{+} / \mathrm{CD} 27^{+} / \mathrm{EV}^{-D 68}{ }^{+}\right)$

unclear $[7,18]$. In our study, based on the rhesus monkey model, a formaldehyde-inactivated EV-D68 vaccine exhibited good immunogenicity and elicited of the neutralizing antibodies in two monkeys with a GMT greater than or equal to 31.8 after the second immunization. The protective efficacy of this experimental inactivated vaccine was evaluated in suckling mice, and it was found that maternal antibodies from the vaccine-Immunized mother mice can protect neonates from EV-D68 challenge. Subsequently, we found that the antibodies elicited by the EV-D68 vaccine mainly recognized the EV-D68 VP1 protein. This phenomenon is consistent with the results of our previous study, in which VP1-specific mAbs were isolated from EV-D68-infected monkeys [16]. Therefore, the immune response induced by from monkeys were sorted into each well of 96-well plates containing $20 \mu \mathrm{l}$ of cell lysis buffer. (a) Sample from monkey no. 15239. (b) Sample from monkey no. 15083

this experimentally inactivated EV-D68 vaccine displayed a similar antigen-presenting preference for the VP1 protein. Similar results have been reported in the development of other inactivated vaccines for picornaviruses, including EV-A71 and coxsackievirus A16(CV-A16) [24-26]. Notably, it was also reported that formaldehyde as an inactivating agent has a limited ability to maintain the structural integrity of antigens from these viruses $[27,28]$, which may result in the failure to induce antibodies covering all the major structural proteins of some viruses [28]. Vaccination can lead to the induction of different types of monoclonal antibodies in vivo by a variety of viral antigens $[29,30]$, and the specific $\mathrm{Nab}$ titer is the main reference for assessing the preventive efficacy of the vaccine in most virus-based vaccines [31-33]. 

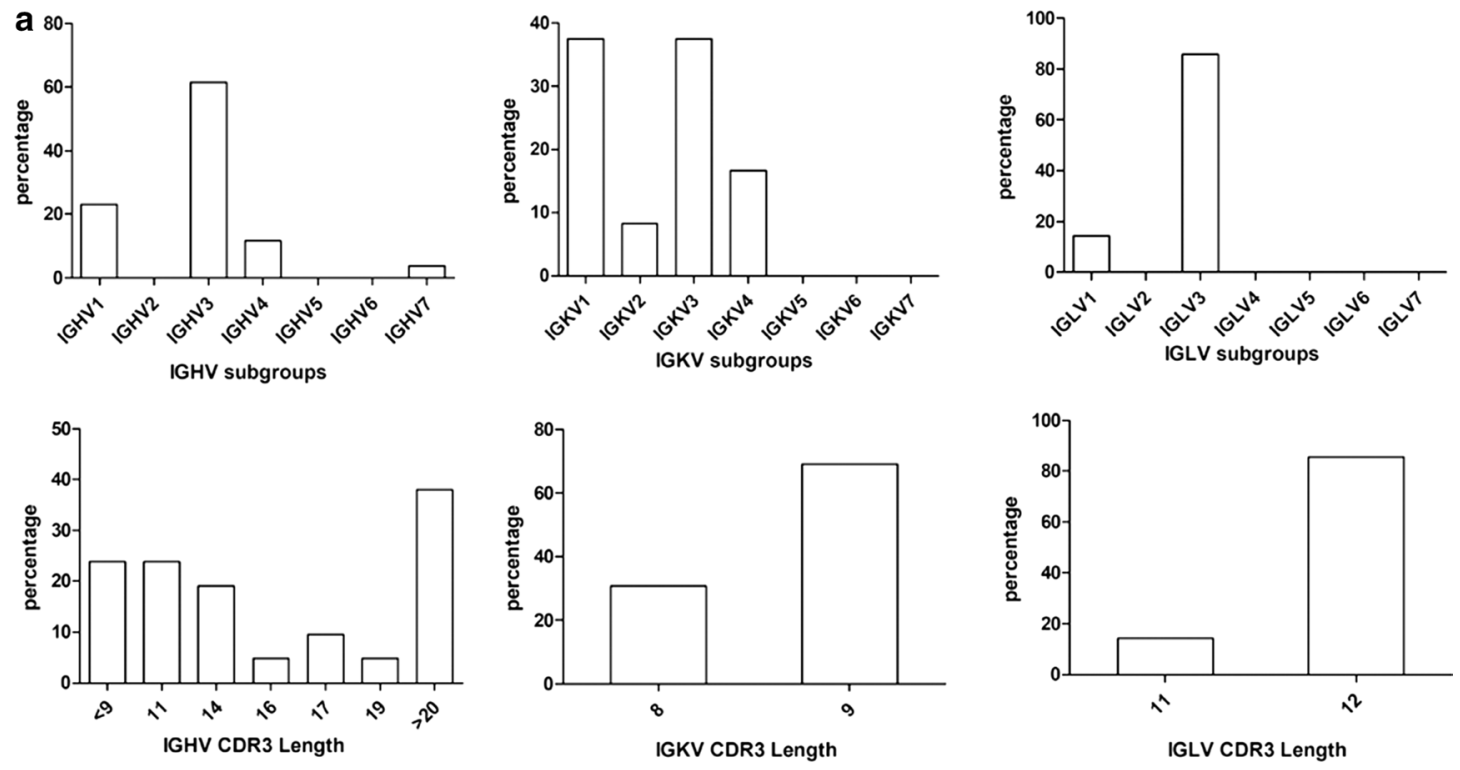

b

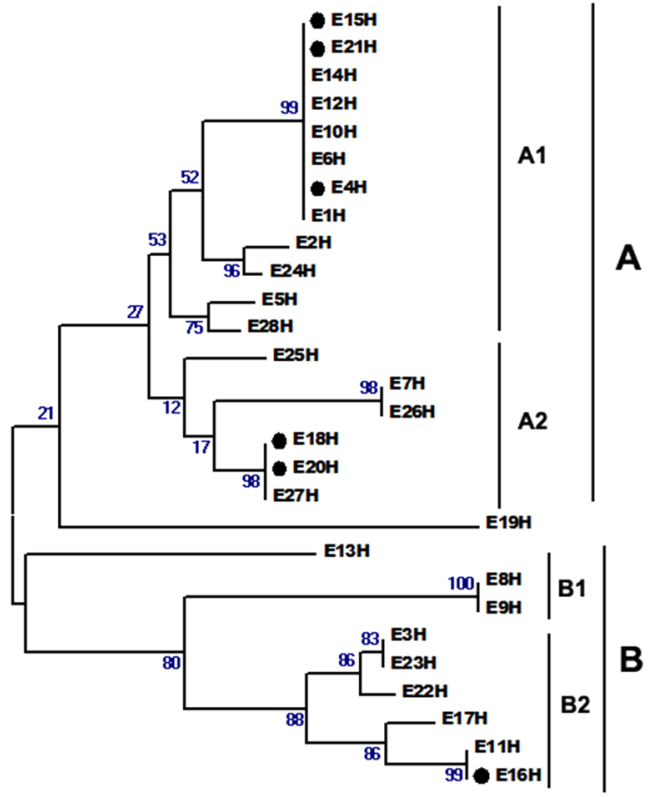

0.1

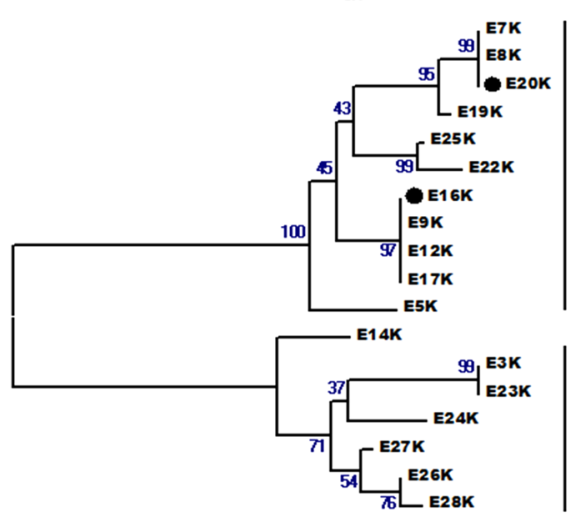

A

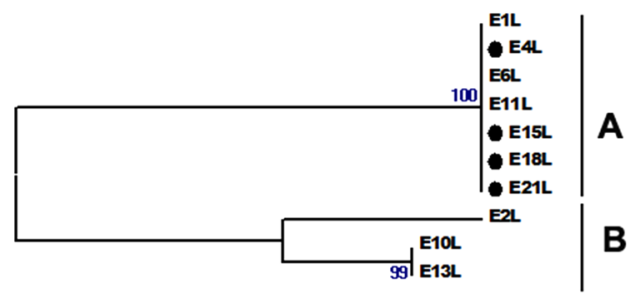

B

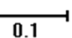

$\lcm{0.1}$ 
४Fig. 4 IGHV, IGKV, IGLV and CDR3 sequencing for clonotype clustering. (a) Germline distribution and CDR3 length of 28 paired mAbs. The $x$-axis represents the different subgroups. The $y$-axis represents the percentage of each subgroup. (b) Analysis of the genetic evolution of the 28 paired mAbs. Sequences were acquired from 28 heavy chains, 18 kappa chains, and 10 lambda chains

However, the Nab titer alone does not completely reflect the effect of various antibodies in the body [30,34]. Therefore, the binding activity and targeting epitopes of mAbs need to be examined in vivo after vaccination to provide valuable information for specific antibody screening and vaccine development $[16,35,36]$. To predict the antibody immune response to vaccination, we examined the phylogenetic relationships, binding activity, and neutralization activity of EVD68-specific mAbs. After immunization of monkeys with EV-D68 vaccine, an IFN- $\gamma$ response and an EV-D68-specific IgG-secreting B-cell response were induced by the EV-D68 whole virus and the EV-D68 VP1 protein. Furthermore, we selected 28 days after primary immunization as the time point for sorting single EV-D68-specific B cells, and we found that the proportion of mAbs with a relatively long CDR3 region (>19 aa) increased, which may be related to antibody specificity [37]. Sequence analysis revealed that the inactivated vaccine elicited antibodies originating from different evolutionary lineages. Other studies have also reported that viral vaccines, such as dengue vaccines and influenza virus vaccines, can elicit multiple types of antibody responses [38, 39].

Effective antiviral vaccine-induced antibodies act in combination with in vivo targeting of viral antigens [40, 41]. The binding and neutralization characteristics of the antibodies were evaluated in our study. We found that the antibodies from clusters A1 and A2 could identify the DE and GH loops of the VP1 protein. Previous studies on EV-D68-neutralizing antibodies have shown that EV-D68-specific monoclonal antibodies can exhibit neutralizing effects by binding the VP1 DE loop region [16]. Here, we found that, of the threecluster A1 mAbs, E15 showed a better binding capacity than
E4 and E21 for the DE loop; however, E15 showed weak binding to the $\mathrm{BC}$ loop. It has been reported that inactivation by formaldehyde treatment can have an undesirable effect on the antigenicity and efficacy of vaccines. For instance, a formaldehyde inactivation of a whole-H1N1 vaccine can reduce the induction of mAbs against conserved epitopes of influenza virus [42]. Another example is that poliovirus inactivated by formaldehyde for 12 days retained the ability to bind to antigen epitopes, but with less efficiency than live virus [43]. The BC loop, a component of the epitopes on EV-D68 surface antigens, does not maintain its structural integrity after 7 days of formaldehyde inactivation, which may result in failure to induce antibodies covering the $\mathrm{BC}$ loop region [19]. In addition to the antibodies that form cluster A, we found that cluster B antibodies exhibited weak binding to the three main structural regions, namely, VP1, VP2 and VP3.

In the present study, we found that mAbs targeting the $\mathrm{GH}$ and DE loops showed neutralizing effects in vitro, and this was also observed in our previous studies [16]. Recently, researchers have found that anti-EV-D68 monoclonal antibodies can bind to the BC, DE, EF and HI loops [44]. Similar to previous studies of mAbs against EV-D68, we found synergistic effects among different mAbs. Using a combination of these antibodies as a cocktail, we found evidence of cooperative activity among mAbs from different lineages. However, whether this activity is a common phenomenon among anti-EV-D68 antibodies still needs to be investigated.

In summary, a variety of antibodies participate in the immune response after inoculation with the inactivated EV-D68 vaccine. In this study, we examined antibody profiles in response to experimental vaccination of rhesus monkeys with an inactivated EV-D68 vaccine and obtained valuable information concerning antibody maturation in response to EV-D68 vaccination or infection. More importantly, compared with the traditional vaccine evaluation strategy, the use of B cell sorting and characterization of specific mAbs provides a new concept for evaluating the immunogenicity of vaccines. 

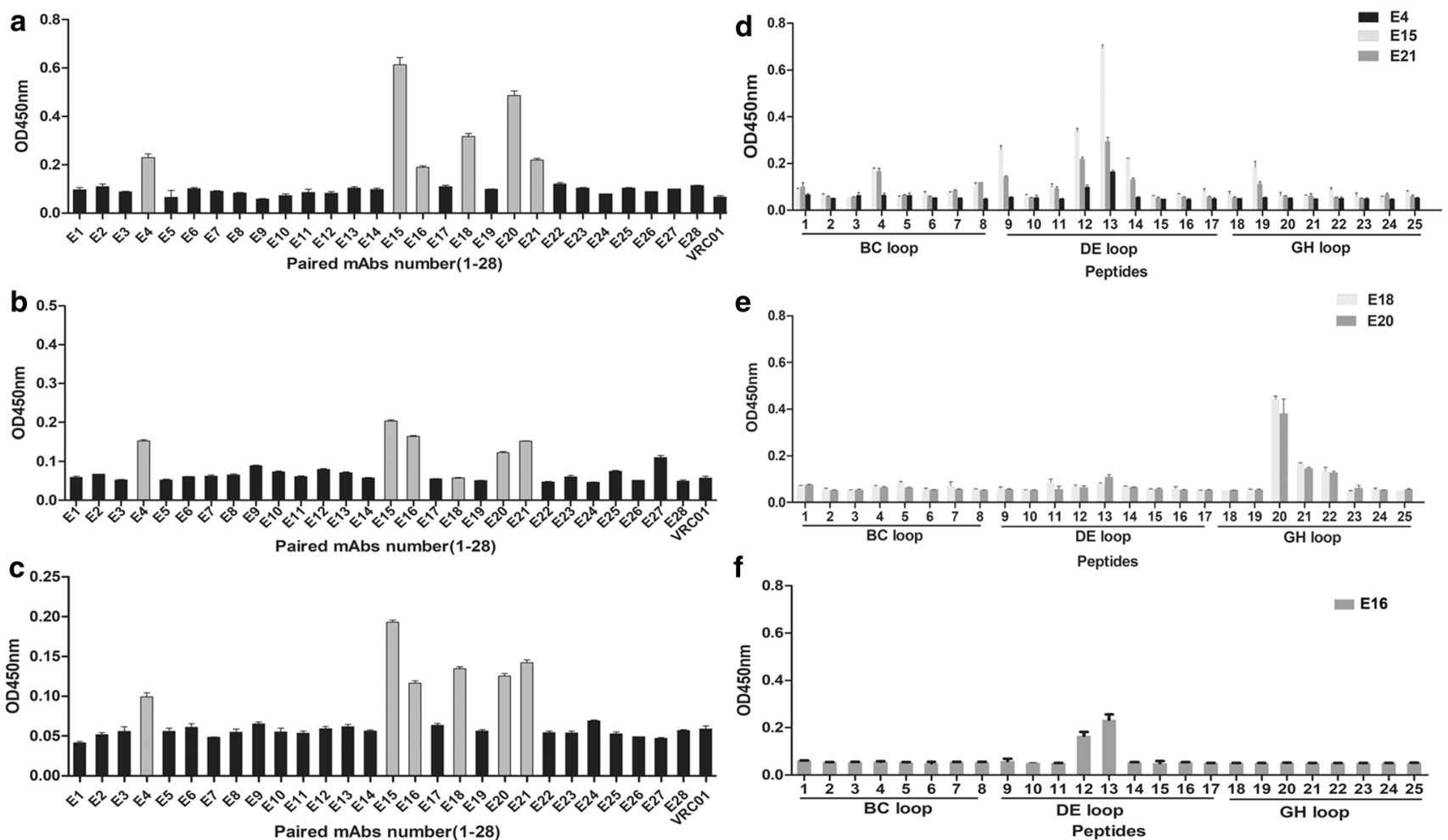

Fig. 5 Binding properties of different mAbs. (a-c) Binding capacity of mAbs against VP1 (a), VP2 (b), and VP3 (c). Purified EV-D68 VP1, VP2, and VP3 proteins were coated onto 96-well plates overnight at $4{ }^{\circ} \mathrm{C}$. VRC01 was used as an irrelevant mAb control. The absorbance at $450 \mathrm{~nm}$ was measured, and the positive sample values were defined as $\mathrm{OD}_{450}>0.1$ and $\mathrm{OD}_{450}>2.1$ times the negative control. (d-f) Binding capacity of mAbs recognizing the DE, BC, and GH

loop regions. The eight critical peptides (peptides 1-8 on the $x$-axis) of the BC loop, nine critical peptides (peptides 9-17 on the $x$-axis) of the DE loop, and eight critical peptides (peptides 18-25 on the $x$-axis) of the GH loop were screened for reactivity with the 28 paired antibodies by ELISA. The mAb-binding capacity is indicated by the $\mathrm{OD}_{450}$ value. (d) mAbs E4, E15, and E21. (e) mAbs E18 and E20. (f) E16
Fig. 6 Neutralization activity and binding activity of antibodies against EV-D68. (a) Neutralization of the EV-D68 KM strain by E15, E18 and E20 (b) Neutralization of the EV-D68 Fermon strain by E15, E18 and E20. The $y$-axis of (a-b) represents the neutralization efficiency. The neutralization efficiency of each mAb was calculated as shown in Materials and methods. (c) $\mathrm{EC}_{50}$ of E15, E18 and E20 for binding to the KM strain EV-D68 VP1. (d) $\mathrm{EC}_{50}$ of E15, E18 and E20 for binding to the Fermon strain EV-D68 VP1
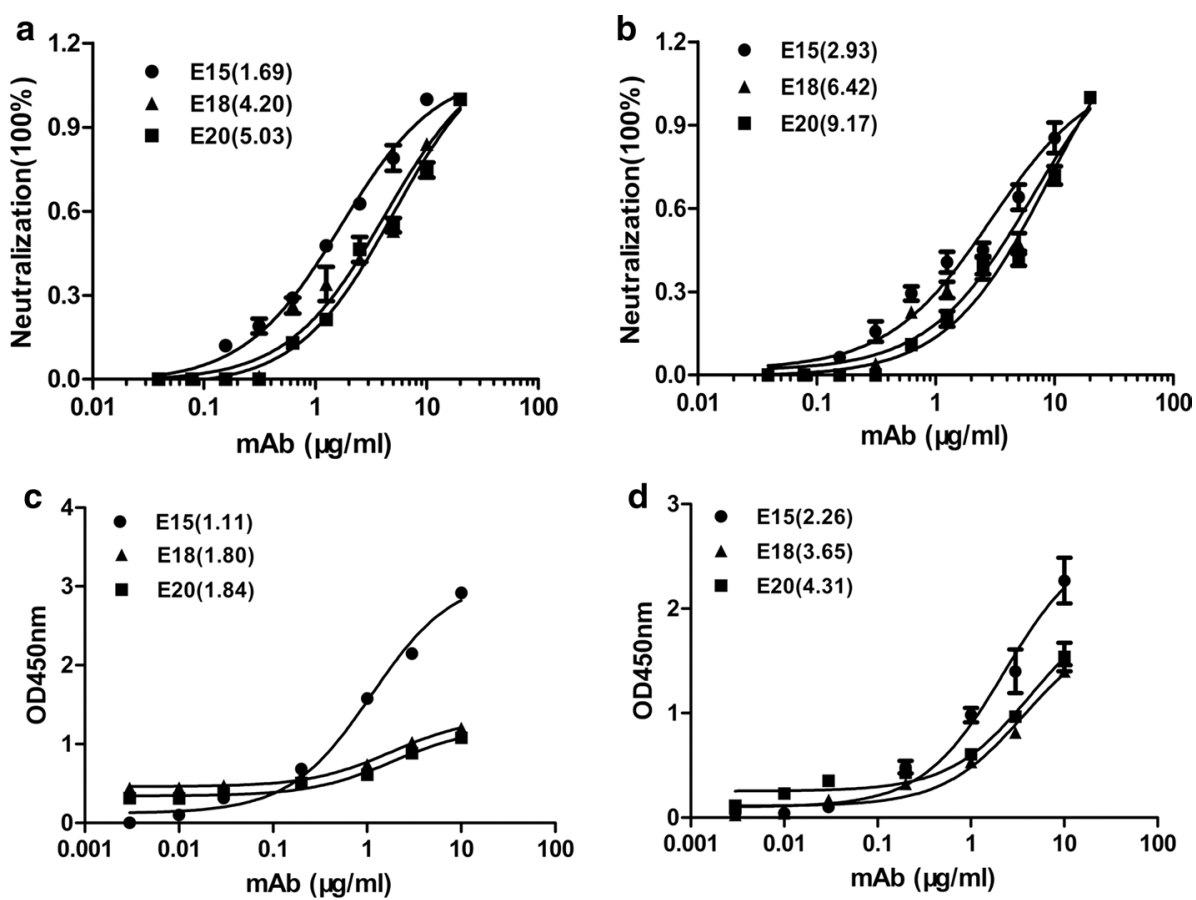
Table 1 The Neutralization potency of the mAbs against EV-D68
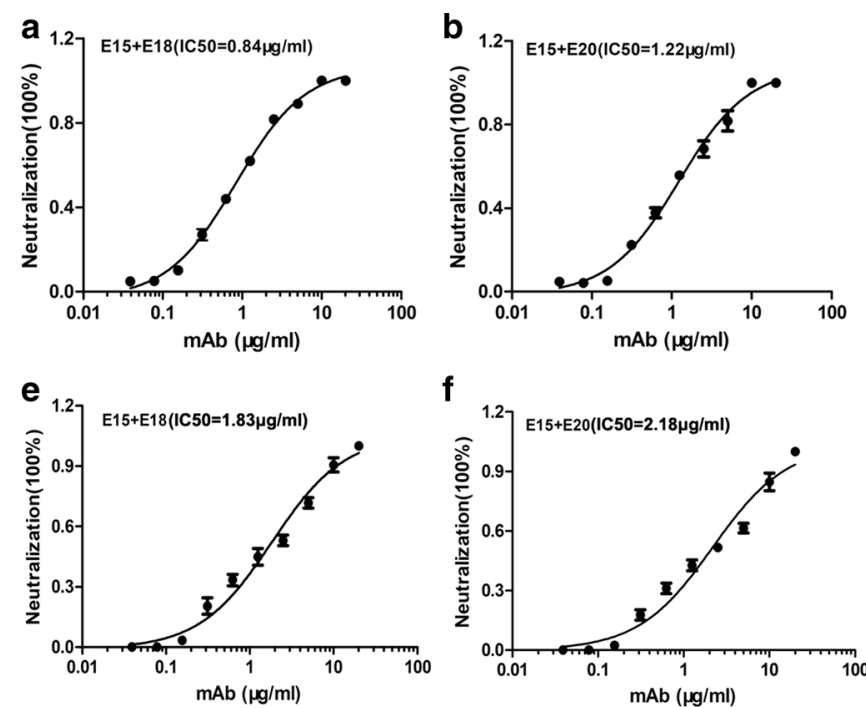

Fig. 7 Paired antibodies show cooperative neutralization. This set of graphs represents one of three separate tests. (a and b) E15 neutralization titers $\left(\mathrm{IC}_{50}\right)$ against $100 \mathrm{CCID}_{50}$ of EV-D68 (KM strain) in the presence of a fixed concentration $(20 \mu \mathrm{g} / \mathrm{ml})$ of E18 (a) and E20 (b). (c and d) E15 neutralization titers $\left(\mathrm{IC}_{50}\right)$ against $100 \mathrm{CCID}_{50}$ of EV-D68 (KM strain) in the presence of the original $\mathrm{IC}_{50}$ concentration $(4.2 \mu \mathrm{g} / \mathrm{ml})$ of E18(c) and the original $\mathrm{IC}_{50}$ concentration $(5.0 \mu \mathrm{g} /$ $\mathrm{ml})$ of E20 (d). (e and f) E15 neutralization titers $\left(\mathrm{IC}_{50}\right)$ against 100 CCID $_{50}$ of EV-D68 (Fermon strain) in the presence of a fixed concentration $(20 \mu \mathrm{g} / \mathrm{ml})$ of E18(e) and E20 (f). (g and h) E15 neutraliza-

Funding This work was supported by the CAMS Innovation Fund for Medical Sciences (2016-I2M-1-014), Basic Scientific Research Funding of National Colleges and Universities (3332020066), and Yunnan
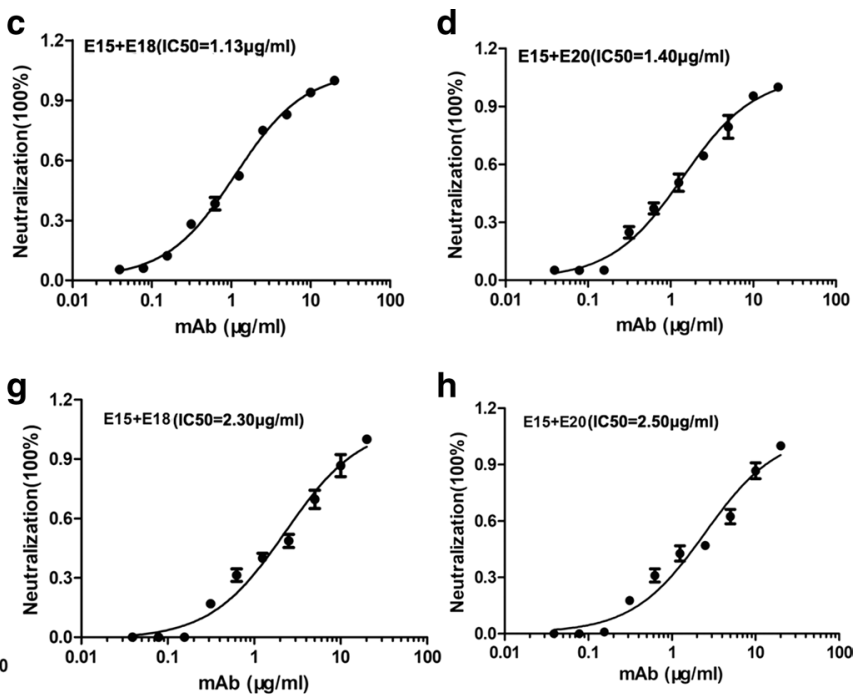

tion titers $\left(\mathrm{IC}_{50}\right)$ against $100 \mathrm{CCID}_{50}$ of EV-D68 (Fermon strain) in the presence of the original $\mathrm{IC}_{50}$ concentration $(6.4 \mu \mathrm{g} / \mathrm{ml})$ of E18 $(\mathrm{g})$ and the original $\mathrm{IC}_{50}$ concentration $(9.2 \mu \mathrm{g} / \mathrm{ml})$ of E20 (h). The $\mathrm{IC}_{50}$ value in parentheses represents the cooperative neutralization of the antibody pair (E15/E18 or E15/E20). The $y$-axis represents the synergistic neutralization efficiency. The synergistic neutralization efficiency of each combination of two mAbs was calculated as described in Materials and methods. $\mathrm{IC}_{50}$ values were calculated using GraphPad Prism 5

Province Major Project of China (2018IA044). The funders had no role in the study design, data collection and analysis, decision to publish, or preparation of the manuscript. 


\section{Compliance with ethical standards}

Conflict of interest All authors declare that the have no competing interest.

\section{References}

1. Imamura T, Oshitani H (2015) Global reemergence of enterovirus D68 as an important pathogen for acute respiratory infections. Rev Med Virol 25(2):102-114

2. Messacar K et al (2015) A cluster of acute flaccid paralysis and cranial nerve dysfunction temporally associated with an outbreak of enterovirus D68 in children in Colorado, USA. Lancet 385(9978):1662-1671

3. Pariani E et al (2017) Letter to the editor: need for a European network for enterovirus D68 surveillance after detections of EV-D68 of the new B3 lineage in Sweden and Italy, 2016. Euro Surveill 22:2

4. Lu QB et al (2014) Detection of enterovirus 68 as one of the commonest types of enterovirus found in patients with acute respiratory tract infection in China. J Med Microbiol 63(Pt 3):408-414

5. Fall A et al (2019) Low circulation of subclade A1 enterovirus D68 strains in senegal during 2014 North America Outbreak. Emerg Infect Dis 25(7):1404-1407

6. Maloney JA et al (2015) MRI findings in children with acute flaccid paralysis and cranial nerve dysfunction occurring during the 2014 enterovirus D68 outbreak. Am J Neuroradiol 36(2):245-250

7. Zhang $C$ et al (2018) A mouse model of enterovirus D68 infection for assessment of the efficacy of inactivated vaccine. Viruses 10:2

8. Zhang C et al (2018) Enterovirus D68 virus-like particles expressed in Pichia pastoris potently induce neutralizing antibody responses and confer protection against lethal viral infection in mice. Emerg Microbes Infect 7(1):3

9. Li R et al (2014) An inactivated enterovirus 71 vaccine in healthy children. N Engl J Med 370(9):829-837

10. Sun M et al (2014) Dynamic profiles of neutralizing antibody responses elicited in rhesus monkeys immunized with a combined tetravalent DTaP-Sabin IPV candidate vaccine. Vaccine 32(9):1100-1106

11. Sun M et al (2017) Immune serum from sabin inactivated poliovirus vaccine immunization neutralizes multiple individual wild and vaccine-derived polioviruses. Clin Infect Dis 64(10):1317-1325

12. Henry Dunand CJ et al (2016) Both neutralizing and non-neutralizing human H7N9 influenza vaccine-induced monoclonal antibodies confer protection. Cell Host Microbe 19(6):800-813

13. Howell KA et al (2017) Cooperativity enables non-neutralizing antibodies to neutralize ebolavirus. Cell Rep 19(2):413-424

14. Mutsvunguma LZ et al (2019) Identification of multiple potent neutralizing and non-neutralizing antibodies against Epstein-Barr virus gp350 protein with potential for clinical application and as reagents for mapping immunodominant epitopes. Virology 536:1-15

15. Ray K et al (2019) Concurrent exposure of neutralizing and nonneutralizing epitopes on a single HIV-1 envelope structure. Front Immunol 10:1512

16. Zheng $\mathrm{H}$ et al (2018) A novel neutralizing antibody specific to the DE loop of VP1 can inhibit EV-D68 infection in mice. J Immunol 201(9):2557-2569

17. Dong $C$ et al (2011) Immunoprotection elicited by an enterovirus type 71 experimental inactivated vaccine in mice and rhesus monkeys. Vaccine 29(37):6269-6275

18. Dai W et al (2018) A virus-like particle vaccine confers protection against enterovirus D68 lethal challenge in mice. Vaccine 36(5):653-659
19. Liu $\mathrm{Y}$ et al (2015) Structure and inhibition of EV-D68, a virus that causes respiratory illness in children. Science 347(6217):71-74

20. Bootz A et al (2017) Protective capacity of neutralizing and nonneutralizing antibodies against glycoprotein B of cytomegalovirus. PLoS Pathog 13(8):e1006601

21. Morens DM, Folkers GK, Fauci AS (2019) Acute flaccid myelitis: something old and something new. MBio 10:2

22. Montes M et al (2019) Enterovirus D68 causing acute respiratory infection: clinical characteristics and differences with acute respiratory infections associated with enterovirus non-D68. Pediatr Infect Dis J 38(7):687-691

23. Pellegrinelli L, et al (2019) Epidemiologic and molecular study of EVs in hospitalized children with severe acute respiratory infection. Pediatr Infect Dis J

24. Wu CY et al (2019) The mature EV71 virion induced a broadly cross-neutralizing VP1 antibody against subtypes of the EV71 virus. PLoS One 14(1):e0210553

25. $\mathrm{Xu} \mathrm{L} \mathrm{et} \mathrm{al} \mathrm{(2015)} \mathrm{A} \mathrm{broadly} \mathrm{cross-protective} \mathrm{vaccine} \mathrm{presenting}$ the neighboring epitopes within the VP1 GH loop and VP2 EF loop of enterovirus 71. Sci Rep 5:12973

26. Shi $\mathbf{J}$ et al (2013) Identification of conserved neutralizing linear epitopes within the VP1 protein of coxsackievirus A16. Vaccine 31(17):2130-2136

27. Sabbaghi A et al (2019) Inactivation methods for whole influenza vaccine production. Rev Med Virol 20:e2074

28. Kim EH, Han GY, Nguyen H (2017) An adenovirus-vectored influenza vaccine induces durable cross-protective hemagglutinin stalk antibody responses in mice. Viruses 9:8

29. van Schooten J, van Gils MJ (2018) HIV-1 immunogens and strategies to drive antibody responses towards neutralization breadth. Retrovirology 15(1):74

30. Kong R et al (2019) Antibody lineages with vaccine-induced antigen-binding hotspots develop broad HIV neutralization. Cell 178(3):567.e19-584.e19

31. Moodie $\mathrm{Z}$ et al (2018) Neutralizing antibody correlates analysis of tetravalent dengue vaccine efficacy trials in Asia and Latin America. J Infect Dis 217(5):742-753

32. Schaid DJ et al (2017) Heritability of vaccine-induced measles neutralizing antibody titers. Vaccine 35(10):1390-1394

33. Tambyah PA et al (2019) An inactivated enterovirus 71 vaccine is safe and immunogenic in healthy adults: a phase I, double blind, randomized, placebo-controlled, study of two dosages. Vaccine 37(31):4344-4353

34. Liu L et al (2013) Study of the integrated immune response induced by an inactivated EV71 vaccine. PLoS One 8(1):e54451

35. $\mathrm{Ku} \mathrm{Z}$ et al (2015) Single neutralizing monoclonal antibodies targeting the VP1 GH loop of enterovirus 71 inhibit both virus attachment and internalization during viral entry. J Virol 89(23):12084-12095

36. Zhou B et al (2019) A bispecific broadly neutralizing antibody against enterovirus 71 and coxsackievirus A16 with therapeutic potential. Antiviral Res 161:28-35

37. Ichiyoshi Y, Casali P (1994) Analysis of the structural correlates for antibody polyreactivity by multiple reassortments of chimeric human immunoglobulin heavy and light chain V segments. J Exp Med 180(3):885-895

38. Meng W et al (2015) Efficient generation of monoclonal antibodies from single rhesus macaque antibody secreting cells. MAbs 7(4):707-718

39. Whittle JR et al (2014) Flow cytometry reveals that H5N1 vaccination elicits cross-reactive stem-directed antibodies from multiple Ig heavy-chain lineages. J Virol 88(8):4047-4057

40. Mankowski MC et al (2018) Synergistic anti-HCV broadly neutralizing human monoclonal antibodies with independent mechanisms. Proc Natl Acad Sci U S A 115(1):E82-E91 
41. Huang KA et al (2019) Structure-function analysis of neutralizing antibodies to H7N9 influenza from naturally infected humans. Nat Microbiol 4(2):306-315

42. Sabbaghi A et al (2019) Inactivation methods for whole influenza vaccine production. Rev Med Virol 29(6):e2074

43. Wilton $\mathrm{T}$ et al (2014) Effect of formaldehyde inactivation on poliovirus. J Virol 88(20):11955-11964

44. Zheng Q et al (2019) Atomic structures of enterovirus D68 in complex with two monoclonal antibodies define distinct mechanisms of viral neutralization. Nat Microbiol 4(1):124-133
Publisher's Note Springer Nature remains neutral with regard to jurisdictional claims in published maps and institutional affiliations. 